\title{
12
}

\section{Aboriginal and Torres Strait Islander community wellbeing: identified needs for statistical capacity}

\section{Ray Lovett}

\section{Introduction}

The ability of Aboriginal and Torres Strait Islander nations to inform and influence policy, program decisions and outcomes is heavily reliant on there being appropriate data to inform results and therefore direction. The cost to our nations, and to Australia broadly, of unreliable or inappropriate data in the area of Aboriginal and Torres Strait Islander health and wellbeing means that, at best, we progress little because of uncertainty about the direction in which to proceed. At worst, unreliable or inappropriate data lead to the perpetuation of ineffective policies and programs because our ability to assess their outcomes and effectiveness is limited.

In addition to concerns about the reliability and appropriateness of data, the manner of its collection, manipulation and reporting also causes great consternation among those of us who lament the inability of the questions on which statistical collections are based to reflect our individual and community realities. This need for data to reflect reality is what Walter and Andersen (2013) refer to as 'the cultural framework 
of Indigenous statistics'. At the national statistics office (NSO) level, there is seemingly a difficulty converting concepts into questions that capture meaningful data about important constructs that give Aboriginal and Torres Strait Islander lives meaning and value, despite having Indigenous advisory structures. The result is a large 'evidence gap' (see Walter, this volume).

Aboriginal and Torres Strait Islander people have observed the inability of NSOs (despite the advice) to progress in this area and have become disengaged or distrustful (Yu 2012). This concern is the likely result of previous experience in research broadly, such as concerns over who controls the process of question (data) development, sampling, data collection, data analysis, data interpretation (context) and reporting of those data. Calls for 'indigenous data sovereignty' stem from these historical legacies and point to a future where indigenous polities maintain, control and protect their data and resulting intellectual property (FNIGC 2007; UN 2007).

Some groups - both domestically and internationally - have turned to their own approaches in progressing what NSOs have been unable to do (Taylor et al. 2012; Nguyen \& Cairney 2013). With this movement has come the assertion of data sovereignty (FNIGC 2007).

The primary way data sovereignty will be achieved in Australian Aboriginal and Torres Strait Islander health and wellbeing policy, program development and review processes is to have the statistical capacity within our population to build these data and to then better inform direction. In addition, we need to connect with non-Indigenous people with statistical capacity who are aware of the current concerns about the statistical construct of our lives and how some analyses are currently being conducted and reported to our detriment.

Unfortunately, there are no readily available data on how many Aboriginal and Torres Strait Islander people have the capacity to undertake statistical analysis and reporting, but proxy estimates suggest the situation is poor. While the focus of this chapter is on health and wellbeing statistics, it is important to recognise that statistical capacity and literacy within the Aboriginal and Torres Strait Islander population are required across all areas of social policy and analysis. Having said that, the building of statistical capacity is also a priority area of need for Australia more broadly (Goldacre 2011; BCA 2015). 
This chapter is presented in two parts. The first part provides a brief historical overview of statistical collections of the Aboriginal and Torres Strait Islander peoples in Australia and then discusses why statistical capacity is important from an Indigenous perspectivespecifically for the realisation of data sovereignty. It concludes by outlining what we currently know about this capacity. Part two provides an overview of current initiatives and approaches that are aimed at improving Indigenous statistical capacity. The chapter concludes with a proposed model for building statistical capacity via research processes using the first national longitudinal study of Aboriginal and Torres Strait Islander wellbeing: Mayi Kuwayu.

\section{Statistical subject or the subject of statistics}

The Commonwealth of Australia Constitution Act 1901 mentions Aboriginal people in section 127: 'In reckoning the numbers of the people of the Commonwealth, or of a State or other part of the Commonwealth, aboriginal natives shall not be counted.' Despite the exclusion of 'full-blood' Aboriginal and Torres Strait Islander people from the Commonwealth census-based count of the Australian population, the states had been collecting or planning to collect data about the Aboriginal populations resident within them from the 1830s onwards (Cannon \& MacFarlane 1982; Briscoe \& Smith 2011).

The inclusion of Aboriginal and Torres Strait Islander people in census counting was one of the primary reasons for the Constitution Alteration (Aboriginals) Act 1967 (the 1967 referendum). Due to the resulting changes to the constitution, Aboriginal and Torres Strait Islander people have been included in the Australian census as a self-identified population from 1971 (CBCS 1972). The counting of Indigenous people in Australia has since flowed through to many other government administrative data collections including hospitals (AIHW 2011a), death and cancer registers (AIHW 2015) and immunisation registers (Centre for Indigenous Health 2004), among others, with a guideline produced to assist (AIHW 2010). More recent developments have included pathology and infectious disease notifications.

These collections are important for the same reasons they are important for the entire population-for example, in planning services and in developing policy. But there have been and continue 
to be complex issues with administrative data that are influenced by a wide range of factors such as systematic racism and a lack of indices reflecting factors that are important to Aboriginal and Torres Strait Islander people (Paradies et al. 2008; Walter \& Andersen 2013). The Australian Bureau of Statistics (ABS) has examined factors affecting reporting of Indigenous status in statistical collections using focus groups. It found that the reliability of Aboriginal and Torres Strait Islander data was negatively affected by the purpose of the data collection, who is conducting it (researchers, government or community organisation) and the mode of collection (ABS 2012). These focus groups also identified that Aboriginal and Torres Strait Islander peoples are less likely to participate in studies and data collections if the data are utilised to create a homogenous Indigenous population and where the analysis portrays Indigeneity as problematic in the manner described by Fforde et al. (2013).

The political, media and social climates can be significant factors in the reliability of Indigenous administrative data. A recent workshop on factors influencing Aboriginal and Torres Strait Islander identification in administrative data discussed how key national events might shape changes in identification:

Positive events that may have influenced the number of people identifying as Indigenous were the Mabo High Court decision in 1992 and the National Apology to the Stolen Generations in 2008. Negative events that seemed to have some influence include the Northern Territory Emergency Response that was rolled out in 2007. Concerns were raised about the next census and the negative symbolism of the current events in Western Australia with the proposed forced closure of many Aboriginal communities. The effects of these events are amplified through media coverage. Participants noted that media can be a barrier to identification by reinforcing internalised racism but also as strength when positive stories are told well. (Nous Group 2015: 2)

\section{Indigenous national engagement with statistical agencies}

Both the ABS and the Australian Institute of Health and Welfare (AIHW) have Indigenous engagement processes for their statistical collections. The ABS has an Aboriginal and Torres Strait Islander Demographic Statistics Expert Advisory Group, which has been tasked 
with providing technical advice and guidance on methodological issues relating to the ABS program of Aboriginal and Torres Strait Islander demographic statistics and advising on communication and engagement strategies (ABS 2011). The most recent meeting notes available online from this group are for December 2011. On examination of the appointment requirements for the advisory group, participants must have 'knowledge of demographic statistics, in particular their technical expertise'. This appears to be limiting given what we know about this expertise among the Aboriginal and Torres Strait Islander population, particularly where it also states that appointees are to have 'knowledge and understanding of the culture and needs of Aboriginal and Torres Strait Islander Peoples' (ABS 2011). It is hard to assess whether the advisory group is meeting its aims, as there is no reference to whom it is advising or whether this advice is being taken up.

The AIHW, in conjunction with the ABS, also administers the National Advisory Group on Aboriginal and Torres Strait Islander Health Information and Data (NAGATSIHID). The main role of NAGATSIHID is to provide strategic advice to the Australian Health Ministers Advisory Council (AHMAC) on Indigenous health data issues. NAGATSIHID has specific responsibility to 'advise and advocate on improving the quality of Indigenous health information and advise on the use of Indigenous health information' (AIHW 2011b). One of the highlighted features of NAGATSIHID is that it has majority Aboriginal and Torres Strait Islander membership drawn from across the different fields of research and teaching, service provision and policy. While an AHMAC member chairs the group, for any decisions, an Indigenous quorum needs to be present (AIHW 2011b).

Common to both structures is the somewhat limiting ability to 'advise', not direct. In addition, the accountability mechanisms for advice provided by membership of both structures are not detailed. While noting the inherent problem of limited statistical capacity, neither structure allows Aboriginal or Torres Strait Islander chairmanship of the advisory structure despite there being greater capacity now than at any time before. Therein lies the problem: with greater Indigenous engagement in these advisory structures comes the advice regarding what Walter and Andersen (2013) call 'the guiding quantitative methodology', and this often conflicts with existing statistical frameworks. Having Aboriginal and Torres Strait Islander voices in 
the discourse surrounding statistical methodologies now, more than at any time before, has the potential to cause conflict or improve the path forward, depending on your view.

\section{The cultural context of health and wellbeing statistics}

There are two main reasons for communicating to Aboriginal and Torres Strait Islander people why statistical capacity is important in our population. The first concerns the current data from the Overcoming Indigenous Disadvantage (OID) report, which tell us that only one indicator is improving and, overall, 'the gap' is not closing (SCRGSP 2014). The health and wellbeing of Aboriginal and Torres Strait Islander peoples continue to be the poorest in Australia (AIHW 2014). Despite this, and despite the consequences of two centuries of colonisation, Aboriginal culture and values remain strong; yet this fact would not be known from reading the OID report. These strengths are considered to be just as important and significant to Aboriginal and Torres Strait Islander people, and they are what should be influencing the statistical agenda (see Bishop, this volume).

Thus, a major barrier to the effective measurement of Aboriginal and Torres Strait Islander health and wellbeing is the lack of a relevant evidence base for factors that Aboriginal and Torres Strait Islander peoples themselves consider important, resulting in the application to data collection of underlying assumptions that other Australians apply to Aboriginal and Torres Strait Islander people. Moreover, there is a lack of integration into data analysis of culture, cultural practices and experiences. Features of the cultural landscape and Aboriginal experience that are highlighted as negatively impacting on wellbeing include exposure to racism, exclusion, marginalisation and negative identity formation (Daniel et al. 2011). The limited available data indicate that there may be relationships between 'on country' practices and risk factors, and that people with a 'strong' sense of identity and higher levels of attachment to culture are happier and display better mental health (Dockery 2011). Hence, interventions devised on the basis of standard evidence lack integration with evidence regarding 
key dimensions that are central to Aboriginal and Torres Strait Islander health and wellbeing - dimensions that are critical to the effectiveness and acceptability of data.

The problem, then, is not so much with gap analysis in and of itself, but rather how we measure and collect data on gaps. We accept that we need to understand what is sustaining the lack of change in key outcomes such as education and employment (upstream indicators) and headline indicators such as life expectancy, but there remain a number of key systemic limitations to the existing framework. In particular, there appears to be an unwillingness to move to more distal levels of measurement and analysis. We seem content to know that we are not reaching equality in educational outcomes, for example, but are unwilling to find out what distal factors may be contributing. These factors are acknowledged in policy reports (Australian Government 2013: 9; SCRGSP 2014: 85), but there appears to be no movement on how these 'data gaps' might be resolved. The fear might be that policies need to focus on the very things current approaches are avoiding: social and cultural differences. What of the distal indicator of a strong connection to mob and country showing a positive correlation with reductions in cardiovascular disease outcomes (Rowley et al. 2008)? Based on this finding and from this perspective, the policy shift would surely need to be cultural strengthening not closure of communities. Local and international literature on the subject of Aboriginal and Torres Strait Islander, Māori and other First Nations groups propose measures that are consistent with indigenous conceptions of wellbeing. These conceptions include:

- relationships with country, spirituality and rituals (Assembly of First Nations 2002; Burgess et al. 2008; Ganesharajah 2009; Prout 2011; Knibb-Lamouche 2012)

- identity and identity representation and racism (Chandler et al. 2003; Henry et al. 2004; Hallett et al. 2007; Paradies et al. 2008; Reading \& Wien 2009; Cunningham \& Paradies 2012; Fforde et al. 2013; Zubrick et al. 2014)

- heritage and language (Chandler et al. 2003; Hallett et al. 2007; Reading \& Wien 2009)

- agency, self-determination, empowerment, fate and control (Hallett et al. 2007; Reading \& Wien 2009; Larsen et al. 2010; KnibbLamouche 2012; Taylor et al. 2012) 
- cultural continuity (Assembly of First Nations 2002; Chandler et al. 2003; Reading \& Wien 2009; Knibb-Lamouche 2012).

These themes address many of the current concerns that indigenous peoples have with contemporary epidemiological approaches to illness measurement in that they are mostly positively focused and are applicable at the local community level as well as at individual and national levels.

The 2008 National Aboriginal and Torres Strait Islander Social Survey (NATSISS) included Aboriginal and Torres Strait Islander community and expert workshops to inform indicators of community wellbeing included in the final survey. To date, only one analysis has examined the 2008 NATSISS data on the relationship between these 'holistic' measures and wellbeing (Dockery 2011), with the results showing that greater participation in cultural events and activities was associated with better mental wellbeing.

There were differences between the results when analysed by rurality in that the positive affects of cultural identity, language use and traditional economic activities accrued mostly within remote areas. Associations between these attributes and greater psychological distress appeared to apply only in nonremote areas. It is hypothesised that this is related to the notion of 'living between cultures' (Dockery 2011: 14) and further evidence of this phenomenon is evident through experiences of racism. Both nonremote and remote groups reported similar rates of exposure to racism overall, but the stronger a nonremote Aboriginal person's identity became, the more likely they were to experience racism in the preceding 12 months (up to 41 per cent) (Dockery 2011).

This work is the first in Australia to empirically demonstrate that Indigenous culture 'should be maintained and leveraged as a solution to Aboriginal and Torres Strait Islander disadvantage, rather than being seen as the problem' (Dockery 2011: 3). It therefore supports the view that Aboriginal and Torres Strait Islander people must be afforded influence over the statistical agenda and, if this is to be revised, improved and managed in a way that is consistent with Aboriginal and Torres Strait Islander values, Aboriginal and Torres Strait Islander people need to be front and centre in any related decision-making process. A related need is to enhance Indigenous statistical capacity. 


\section{What do we mean by Indigenous statistical capacity?}

Statistics is a form of mathematical analysis involving the use of quantified representations, models and summaries for a given set of empirical data or real-world observations. Statistical analysis involves the process of collecting and analysing data and then summarising the data into numerical format. There are two elements to statistical capacity. The first is having the relevant training in methods and approaches to appropriately inform the compilation of statistics. The second concerns the 'frame of view' used in preparing the questions we seek to answer; this also invariably informs the way we analyse and report data - the way we give it meaning (Walter \& Andersen 2013; Walter, this volume). This second aspect is critical to understanding how we engage in the measurement of Aboriginal and Torres Strait Islander health and wellbeing in Australia.

Capacity describes an ability to do something. In this sense, then, Aboriginal and Torres Strait Islander statistical capacity is the ability of Aboriginal and Torres Strait Islander people to perform mathematical analysis involving the use of quantified representations, models and summaries for a given set of empirical data or real-world observations within a frame of view that gives the data meaning to our nations and peoples. This frame of view constitutes how the world around us is connected. For Aboriginal people, this includes the centrality of family connections (mob), our connection to country or countries and the stories that maintain those links with family and country. Family and country are crucial as, without these, connection is limited or lost. Importantly, these elements endure across the country and across the statistical classifications of remote, regional and urban. Operationalising these concepts requires those with this frame of view to be at the forefront of question design and analysis.

\section{School-based statistical capacity}

The foundation of statistics is mathematics. Every three years Australian students participate in the Programme for International Student Assessment (PISA), which measures three educational outcomes - literacy in: mathematics, science and reading. In 2012, about 14,500 Australian 15-year-olds participated in PISA, including 
1,991 Indigenous students from across urban, regional and remote settings. The 2012 PISA results for mathematical literacy indicated that Indigenous students were more than 2.5 years behind their non-Indigenous peers and that these results were particularly stark compared with those for reading and science (Dreise \& Thomson 2014). Consistently, we see education outcome reports identify a growing inequality of educational outcomes between Indigenous and other students as they move through the school years; and this gap has been growing for some time (Mellor \& Corrigan 2004).

Previous research has identified that methods of teaching primary mathematics can be ineffective for Aboriginal students because they are not related to their world and everyday experiences (Matthews et al. 2007). This results in alienation from maths in the later years of primary school (Matthews et al. 2003). It is encouraging, then, to see an increasing use of more novel approaches to primary and secondary school teaching of maths (AAMT 2015). This includes projects such as 'Maths as Story Telling' (MAST), a teaching approach designed to assist Indigenous students in their understanding of algebra through the creation and manipulation of their own symbols for equations (Matthews et al. 2007; Ewing et al. 2010). Coincidentally, issues with data integrity have meant results have not been released, although some sites have reported positive outcomes at the student and school levels (AAMT 2013).

There is also a range of other high school statistical and mathematics programs that are designed to engage students in statistics. These include the Statistical Society of Australia National Secondary Schools Poster Competition and the Commonwealth Scientific and Industrial Research Organisation Mathematics in Schools project (CSIRO 2014; SSA 2014). While these programs are available, there are no data on their uptake by Aboriginal and Torres Strait Islander students or by specific schools.

\section{Tertiary and further education-based statistical capacity}

In the vocational education and training (VET) sector, statistical training is usually embedded within broader mathematics programs (TAFE NSW 2015). These are generalist programs and provide the basic requirements of mathematics for statistical concepts and are relatively 
common across Australia. Data about the number of Aboriginal and Torres Strait Islander students enrolled in and completing these courses (and units) are limited due to reliability issues. As for the university sector, some courses at the undergraduate level teach research methods including quantitative analysis (sociology, economics and psychology, for example), however, access to data concerning Aboriginal and Torres Strait Islander enrolment and completions requires a specific request to the Department of Education (only aggregated broadly themed data are available on their website). Advanced statistics training is undertaken in specialist postgraduate teaching and research programs in the disciplines of epidemiology, public health, biostatistics, demography, econometrics and psychology. These courses are less common throughout the country and access to enrolment data for Aboriginal and Torres Strait Islander students is limited and the data are unreliable due to the variable recording of Indigenous status at enrolment. The Statistical Society of Australia lists seven accredited statistics courses across 10 universities, keeping in mind there are other courses that provide education in statistics (SSA 2015).

\section{A successful higher education model?}

The National Centre of Epidemiology and Population Health (NCEPH) at The Australian National University (ANU) has been running a Field Epidemiology Training Program (FETP) for the past 20 years. The MPhil (Applied Epidemiology), previously the Master of Applied Epidemiology (MAE), is a two-year research degree that emphasises learning-by-doing. The program teaches epidemiology through coursework and learning in a field placement, such as with a health department. The MPhil (Applied Epidemiology) is Australia's only FETP and is part of the international network of Field Training Programs in Epidemiology \& Public Health Interventions Network. The program has been extremely successful both as a field-based training program and for the high proportion of Aboriginal and Torres Strait Islander graduates (about 30 of the total of more than 150 graduates) as a result of introducing a specific Indigenous training commitment in 1998 (Guthrie et al. 2011). In 2010 funding from the Australian Government Department of Health and Ageing, which had been responsible for the growth in Master of Public Health degrees across the country, was withdrawn (Lin et al. 2009). This had 
a severe impact on the MAE as the funds were no longer available to support the students' living expenses (a stipend) and, although the field-based training element remained, the host organisations are now required to find upwards of $\$ 50,000$ each year to host a student. These changes came despite compelling arguments for the program's continuation, including the potential detrimental impact on statistical and epidemiological capacity (Guthrie et al. 2011).

\section{Inclusion of statistical capacity in research programs: Mayi Kuwayu}

As with international examples (Assembly of First Nations 2002; Larsen et al. 2010), the idea for the first national longitudinal study of Aboriginal and Torres Strait Islander wellbeing, Mayi Kuwayu, was born out of concern about the absence of constructs that are important to Aboriginal and Torres Strait Islander peoples' wellbeing in existing administrative data (Lovett et al. 2015). While the study is still in the early development phase, built into the proposal is the establishment of a community-based statistical capacity-building program, which will be developed between three Aboriginal and Torres Strait Islander peak research and community groups and The Australian National University. The program aims to run a residential-based short course in quantitative methods among staff working within Aboriginal and Torres Strait Islander health services. Given the number of these services across the country, the pool of participants is potentially large. The aim is to provide the administrative and Aboriginal and Torres Strait Islander health staff with statistical skills that will enable them to collect, prepare, analyse and report their own service data in ways that are meaningful to their service and the community. This capacitybuilding program was written into the research proposal as a result of the research team seeing community-based organisations, including Aboriginal and Torres Strait Islander health services, struggle with their electronic databases. The opportunity to assist in the building of statistical capacity so that data can be used for advocacy and resourcing enables the research team to meet a need that will have tangible and sustained benefits for individuals and organisations, as required by National Health and Medical Research Council ethical guidelines (NHMRC 2003). 


\section{Conclusion}

There has been slow progress in developing statistics that are conceptualised from an Aboriginal and Torres Strait Islander 'frame of view'. This is likely a result of the poor level of statistical capacity and the restrictive processes of defining wellbeing indicators to date. Statistical capacity has the potential to enhance the development of indices relevant to Aboriginal and Torres Strait Islander people's lives from our frame of view, and to position us at the table to assert data sovereignty. These new data will give Aboriginal and Torres Strait Islander peoples and nations the power to demonstrate to individuals, institutions, communities and governments evidence for the development of policy and programs.

Greater direct engagement in the conceptualisation, design and data collection, analysis and reporting will enable more meaningful information to be provided to policymakers and also enable communities to engage in a circular process whereby they are able to welcome the benefits of data collection and analysis, leading to more open discourse about the information needed to inform the evidence base. To ensure there is enhanced statistical capacity within our nations, mathematics education and statistical training that encompass direct relevance to our world views and ways of being are required. Programs such as the MPhil Epidemiology program and other community-based statistical capacity-building programs have the potential to facilitate statistical capacity and need to be supported. As well as these programs assisting with statistical capacity, they will also help to develop quantitative indicators of wellbeing from an Aboriginal and Torres Strait Islander frame of view. The combination of capacity and frame of view will then influence Indigenous data sovereignty.

\section{References}

Assembly of First Nations (2002). Community health indicators: second year of the project, Institute of the Environment, Ottawa.

Australian Association of Mathematics Teachers (AAMT) (2013). Make it count: numeracy, mathematics and Indigenous learners project summary, AAMT, Adelaide, mic.aamt.edu.au/Resources/Make-ItCount-2009-2012/Publications-and-statements. 
Australian Association of Mathematics Teachers (AAMT) (2015). Make it count: maths and indigenous learners, AAMT, Adelaide, mic.aamt. edu.au/.

Australian Bureau of Statistics (ABS) (2011). Aboriginal and Torres Strait Islander Demographic Statistics Expert Advisory Group terms of reference, ABS, Canberra, abs.gov.au/websitedbs/ c311215.nsf/88e17471717cdbc5ca25778a001d9500/0a906fcb6f13c 76dca257a93001f7bcc/\$FILE/Terms\%20of\%20Reference.pdf.

Australian Bureau of Statistics (ABS) (2012). Perspectives on Aboriginal and Torres Strait Islander identification in selected data collection contexts, Cat. no. 4726.0, ABS, Canberra.

Australian Government (2013). National Aboriginal and Torres Strait Islander health plan (NATSIHP) 2013-2023, Department of Health and Ageing, Canberra.

Australian Institute of Health and Welfare (AIHW) (2010). National best practice guidelines for collecting Indigenous status in health data sets, AIHW, Canberra, aihw.gov.au/WorkArea/DownloadAsset. aspx $? \mathrm{id}=6442458760$.

Australian Institute of Health and Welfare (AIHW) (2011a). The health and welfare of Australia's Aboriginal and Torres Strait Islander people: an overview, 2011, AIHW, Canberra.

Australian Institute of Health and Welfare (AIHW) (2011b). National advisory group on Aboriginal and Torres Strait Islander health information and data: strategic plan 2010-2015, AIHW, Canberra.

Australian Institute of Health and Welfare (AIHW) (2014). Australia's health 2014, AIHW, Canberra.

Australian Institute of Health and Welfare (AIHW) (2015). Australian burden of disease study: fatal burden of disease in Aboriginal and Torres Strait Islander people 2010, Australian Burden of Disease Study, Series 2. Cat. no. BOD 2, AIHW, Canberra.

Biostatisticians Collaboration of Australia (BCA) (2015). About the $B C A$, BCA, Sydney, bca.edu.au/aboutbca.html. 
Briscoe G \& Smith L (2011). Australian Aboriginal census, 1921-1944, Australian Social Science Data Archive, The Australian National University, Canberra, Dataset ID:au.edu.anu.ada.ddi.20002-nsw.

Burgess C, Berry H, Gunthorpe W \& Bailie R (2008). Development and preliminary validation of the 'Caring for Country' questionnaire: measurement of an Indigenous Australian health determinant. International Journal for Equity in Health 7(26).

Cannon M \& MacFarlane I (eds) (1982). The Aborigines of Port Phillip, 1835-1839, Victorian Government Printing Office, Melbourne.

Centre for Indigenous Health (2004). Needs analysis of immunisation for Aboriginal and Torres Strait Islander people in Queensland: general practitioner survey of Indigenous immunisation issues, Centre for Indigenous Health, University of Queensland, Brisbane.

Chandler MJ, Lalonde CE, Sokol B \& Hallett D (2003). Personal persistence, identity development, and suicide: a study of native and non-native North American adolescents, Monographs of the Society for Research in Child Development Vol. 68, Wiley-Blackwell, Boston \& Oxford.

Commonwealth Bureau of Census and Statistics (CBCS) (1972). Official year book of the Commonwealth of Australia, No. 58, CBCS, Canberra.

Commonwealth Scientific and Industrial Research Organisation (CSIRO) (2014). Mathematicians in schools, CSIRO, Canberra, mathematiciansinschools.edu.au/.

Cunningham J \& Paradies YC (2012). Socio-demographic factors and psychological distress in Indigenous and non-Indigenous Australian adults aged 18-64 years: analysis of national survey data. BMC Public Health 12(95), doi:10.1186/1471-2458-12-95.

Daniel M, Lekkas P \& Cargo M (2011). Environments and cardiometabolic diseases in Aboriginal populations. Heart, Lung and Circulation 19(5):306-15, doi:10.1016/j.hlc.2010.01.005.

Dockery AM (2011). Traditional culture and the wellbeing of Indigenous Australians: an analysis of the 2008 NATSISS, CLMR Discussion Paper 2011/01, Centre for Labour Market Research, Curtin Business School, Curtin University, Perth. 
Dreise T \& Thomson S (2014). Unfinished business: PISA shows Indigenous youth are being left behind, ACER Occasional Essay, February 2014, Australian Council for Educational Research, Melbourne.

Ewing B, Cooper T, Baturo A, Matthews C \& Sun H (2010). Contextualising the teaching and learning of measurement within Torres Strait Islander schools. Australian Journal of Indigenous Education 39:11-23.

Fforde C, Bamblett L, Lovett R, Gorringe S \& Fogarty B (2013). Discourse, deficit and identity: Aboriginality, the race paradigm and the language of representation in contemporary Australia. Media International Australia Incorporating Culture and Policy 149:162-73.

First Nations Information Governance Centre (FNIGC) (2007). OCAP: ownership, control, access and possession, sanctioned by the First Nations Information Governance Committee, Assembly of First Nations, National Aboriginal Health Organization, Ottawa.

Ganesharajah C (2009). Indigenous health and wellbeing: the importance of country, Native Title Research Report No. 1/2009, Australian Institute of Aboriginal and Torres Strait Islander Studies, Canberra.

Goldacre B (2011). The statistical error that just keeps on coming. The Guardian, 10 September 2011, theguardian.com/ commentisfree/2011/sep/09/bad-science-research-error.

Guthrie J, Dance P, Kelly P, Lokuge K, McPherson M \& Faulkner S (2011). Public health capacity development through Indigenous involvement in the Master of Applied Epidemiology program: celebrations and commiserations. Australian Aboriginal Studies 2011(2):102-10.

Hallett D, Chandler MJ \& Lalonde CE (2007). Aboriginal language knowledge and youth suicide. Cognitive Development 22:392-9.

Henry BR, Houston S \& Mooney GH (2004). Institutional racism in Australian healthcare: a plea for decency. Medical Journal of Australia 180(10):517-20. 
Knibb-Lamouche J (2012). Culture as a social determinant of health: examples from native communities, Paper prepared for the Roundtable on the Promotion of Health Equity and the Elimination of Health Disparities, Institute on Medicine, Seattle, 14 November 2012.

Larsen JN, Schweitzer P \& Fondahl G (eds) (2010). Arctic social indicators: a follow-up to the Arctic Human Development Report, Nordic Council of Ministers, Copenhagen.

Lin V, Watson R \& Oldenburg B (2009). The future of public health: the importance of workforce. Australia and New Zealand Health Policy 9(6):4, doi:10.1186/1743-8462-6-4.

Lovett R, Banks E, Chapman J \& Strelein L (2015). Development of the first national Aboriginal and Torres Strait Islander longitudinal study of wellbeing: research proposal, Unpublished report, The Australian National University and the Australian Institute of Aboriginal and Torres Strait Islander Studies, Canberra, aiatsis.gov.au/mayikuwayu.

Matthews C, Cooper TJ \& Baturo AR (2007). Creating your own symbols: beginning algebraic thinking with indigenous students, Paper presented at the 31st Annual Conference of the International Group for the Psychology of Mathematics Education, Seoul, 8-13 July 2007, eprints.qut.edu.au/14627/.

Matthews S, Howard P \& Perry JR (2003). Working together to enhance Australian Aboriginal students' mathematics learning. In Bragg L, Campbell C, Herbert G \& Mousley J (eds), Mathematics education research: innovation, networking, opportunity. Proceedings of the 26th Annual Conference of the Mathematics Education Research Group of Australasia, Deakin University, Geelong, 6-10 July 2003, MERGA, Geelong, Vic.

Mellor S \& Corrigan M (2004). The case for change: a review of contemporary research on Indigenous education outcomes, Australian Council for Educational Research, Melbourne, research.acer.edu. $\mathrm{au} / \mathrm{cgi} /$ viewcontent.cgi?article $=1006 \&$ context $=$ aer. 
National Health and Medical Research Council (NHMRC) (2003). Values and ethics: guidelines for ethical conduct in Aboriginal and Torres Strait Islander health research (values and ethics), NHMRC, Canberra.

Nguyen O \& Cairney S (2013). Literature review of the interplay between education, employment, health and wellbeing for Aboriginal and Torres Strait Islander people in remote areas: working towards an Aboriginal and Torres Strait Islander wellbeing framework, CRCREP Working Paper CW013, Ninti One Limited, Alice Springs, NT.

Nous Group (2015). Understanding Aboriginal and Torres Strait Islander identification: draft workshop report, Nous Group, Canberra.

Paradies Y, Harris R \& Anderson I (2008). The impact of racism on Indigenous health in Australia and Aotearoa: towards a research agenda, CRCAH Discussion Paper Series No. 4, Cooperative Research Centre for Aboriginal Health and Flinders University, Adelaide.

Prout S (2011). Indigenous wellbeing frameworks in Australia and the quest for quaification. Social Indicators Research 109(2):317-36.

Reading CL \& Wien F (2009). Health inequalities and social determinants of Aboriginal peoples' health, National Collaborating Centre for Aboriginal Health, Prince George, British Columbia.

Rowley KG, O'Dea K, Anderson I, McDermott R, Saraswati K, Tilmouth R \& Brown A (2008). Lower than expected morbidity and mortality for an Australian Aboriginal population: 10-year follow-up in a decentralised community. Medical Journal of Australia 188(5):283-7.

Statistical Society of Australia (SSA) (2014). SSA national secondary schools poster competition, SSA, Canberra, www.ssaipostercomp. info/:SSAI.

Statistical Society of Australia (SSA) (2015). Accredited university courses in statistics 2015, SSA, Canberra, statsoc.org.au/careersaccreditation/professional-accreditation/accredited-courses/.

Steering Committee for the Review of Government Service Provision (SCRGSP) (2014). Overcoming Indigenous disadvantage: key indicators 2014, Productivity Commission, Canberra. 
TAFE NSW (2015). Mathematics and science for further study, TAFE NSW, Sydney, tafensw.edu.au/course/10222NAT-01V0115WCN-002/mathematics-and-science-for-further-study.

Taylor J, Doran B, Parriman M \& Yu E (2012). Statistics for community governance: the Yawuru Indigenous population survey of Broome, CAEPR Working Paper No. 82/2012, Centre for Aboriginal Economic Policy Research, The Australian National University, Canberra.

United Nations (UN) (2007). United Nations declaration on the rights of indigenous peoples, General Assembly Resolution 61/295, 13 September 2007, United Nations, New York.

Walter M \& Andersen C (2013). Indigenous statistics: a quantitative research methodology, Left Coast Press, Walnut Creek, CA.

Yu P (2012). The power of data in Aboriginal hands, CAEPR Topical Issue 2012/4, Centre for Aboriginal Economic Policy Research, The Australian National University, Canberra, caepr.anu.edu.au/ Publications/topical/2012TI4.php.

Zubrick S, Dudgeon P, Gee G, Glaskin B, Kelly K, Paradies Y \& Walker $R$ (2014). Social determinants of Aboriginal and Torres Strait Islander social and emotional wellbeing. In Dudgeon P, Milroy $\mathrm{H}$ \& Walker R (eds), Working together: Aboriginal and Torres Strait Islander mental health and wellbeing principles and practice, 2nd edn, Telethon Kids Institute, Perth, aboriginal.telethonkids.org.au/ media/699863/Working-Together-Book.pdf. 
This text is taken from Indigenous Data Sovereignty: Toward an agenda, edited by Tahu Kukutai and John Taylor, published 2016 by ANU Press, The Australian National University, Canberra, Australia. 